\section{Biomasse et stocks de carbone en Afrique centrale : importance de l'allométrie des arbres}

Grace Jopaul Loubota PANzou

\section{RÉSUMÉ}

Quantifier les stocks de biomasse et de carbone dans les forêts tropicales est essentiel pour la mise en œuvre des stratégies d'atténuation des changements climatiques, et notamment le mécanisme de réduction des émissions dues à la déforestation et la dégradation des forêts. Les scientifiques s'accordent aujourd'hui à utiliser une équation allométrique de biomasse générale, combinée à une allométrie hauteur-diamètre ajustée sur des données locales non destructives. Or, l'allométrie des arbres varie entre les espèces et stratégies fonctionnelles d'une part, entre les types de forêts et conditions du milieu d'autre part. L'objectif de cette thèse de doctorat était d'étudier les variations de l'allométrie des arbres entre espèces coexistantes, et entre sites et types de forêts, en vue d'améliorer les estimations de la biomasse et des stocks de carbone en Afrique centrale. Les variations des allométries hauteur-diamètre et houppier-diamètre ont été rapportées pour les 45 espèces d'arbres coexistantes d'un site représentatif des forêts semi-décidues à Celtis du nord du Congo. Les relations entre les traits architecturaux dérivés à partir des allométries spécifiques aux espèces et les traits fonctionnels ont mis en évidence un continuum d'espèces entre les espèces de grande taille dominant la canopée et les espèces de petite taille dominant le sous-bois. Les espèces d'arbres de grande taille étaient exigeantes en lumière, dispersées par le vent, décidues avec une large contribution à la biomasse forestière, alors que les espèces d'arbres de petite taille étaient tolérantes à l'ombrage, dispersées par les animaux, sempervirentes et plus abondantes en densité de tiges. Les variations des allométries hauteur-diamètre et houppier-diamètre ont été également montrées entre les types de forêts en Afrique centrale. Ces tendances étaient en outre confirmées pour les espèces communes entre les types de forêts, suggérant un contrôle environnemental sur les variations de l'allométrie des arbres. Des attributs architecturaux dérivés des allométries spécifiques aux sites, en plus des attributs plus classiques de structure et de composition, ont pu être dérivés et reliés aux variations spatiales de la biomasse et des stocks de carbone. Cette thèse de doctorat $a$ fourni de nouvelles données pour les futures cartes de biomasse dans le cadre du suivi du carbone forestier en Afrique centrale.

Mots-clés : allométrie des arbres, hauteur totale, dimensions du houppier, biomasse aérienne, stocks de carbone, espèces coexistantes, type de forêt, traits ou attributs architecturaux, traits fonctionnels, variation spatiale de la biomasse, Afrique centrale.
Biomass and carbon stocks in Central Africa: importance of tree allometry

\section{ABSTRACT}

Quantifying biomass and carbon stocks in tropical forests is essential for the implementation of climate change mitigation strategies, including the mechanism for reducing emissions from deforestation and forest degradation. Scientists have reached agreement on the use of a general allometric biomass equation combined with height-diameter allometry adjusted to local non-destructive data. However, tree allometry varies between species and functional strategies on the one hand, and between forest types and environmental conditions on the other. The aim of this $\mathrm{PhD}$ thesis was to study the variations in tree allometry among coexisting tree species and between sites and forest types, in order to improve estimations of biomass and carbon stocks in Central Africa. At the species scale, variations in height-diameter and crown dimensions-diameter allometries were reported among the 45 coexisting tree species in a representative site of semi-deciduous Celtis forest in northern Congo. The relationships between architectural traits (total height and crown dimensions) derived from species-specific allometries and functional traits highlighted a continuum of species between large-statured canopy species and small-statured understory species. The large-statured species tended to be light-demanding, wind-dispersed, deciduous and major contributors to forest biomass, while the small-statured understory species tended to be shade-tolerant, animal-dispersed, evergreen and most abundant in terms of stem density. At the site scale, we showed the variations in height-diameter allometry between forest types and, for the first time in Central Africa, variations in crown-diameter allometry between forest types. These trends were also confirmed for species common to different forest types, suggesting an environmental control in tree allometry. From the site-specific allometries, architectural attributes (total height and crown dimensions), in addition to the more classic structural attributes (basal area) and composition attributes (wood density), were derived and related to spatial variations in biomass and carbon stocks. This PhD thesis provided new data needed for the future biomass maps that will support forest carbon monitoring in Central Africa.

Keywords: tree allometry, total height, crown size, aboveground biomass, carbon stocks, species coexistence, forest type, architectural traits or attributes, functional traits, spatial variation in biomass, Central Africa.
Biomasa y reservas de carbono en África Central: importancia de la alometría de los árboles

\section{RESUMEN}

Cuantificar las reservas de biomasa y de carbono en los bosques tropicales es esencial para la aplicación de estrategias de atenuación de los cambios climáticos, especialmente para el mecanismo de reducción de las emisiones debidas a la deforestación y la degradación de los bosques. Los científicos están de acuerdo actualmente en utilizar una ecuación alométrica de biomasa general, combinada con una alometría altura-diámetro ajustada a datos locales no destructivos. Sin embargo, la alometría de los árboles varía entre las especies y las estrategias funcionales por un lado, y entre los tipos de bosque y las condiciones del medio, por el otro lado. El objetivo de esta tesis doctoral era estudiar las variaciones de la alometría de los árboles en función de especies coexistentes, y en función de zonas y tipos de bosque, para mejorar las estimaciones de la biomasa y de las reservas de carbono en África Central. Las variaciones de las alometrías altura-diámetro y copa-diámetro se calcularon entre las 45 especies de árboles coexistentes de una zona representativa de los bosques semicaducifolios de Celtis del Congo norte. Las relaciones entre los rasgos arquitectónicos derivados a partir de las alometrías específicas de las especies y los rasgos funcionales pusieron en evidencia un continuo de especies entre las especies de gran altura con dosel forestal dominante y las especies de poca altura que dominan el sotobosque. Las especies de árboles de gran altura requerían mucha luz, eran dispersadas por el viento, caducifolias y tenían una amplia contribución a la biomasa forestal, mientras que las especies de árboles de poca altura toleraban la sombra, eran dispersadas por los animales, perennes y más abundantes en cuanto a la densidad de tallos. Las variaciones de las alometrías altura-diámetro y copa-diámetro se calcularon igualmente entre los tipos de bosque en África Central. Estas tendencias fueron también confirmadas para las especies comunes entre los tipos de bosques, lo que sugirió un control medioambiental sobre las variaciones de la alometría de los árboles. Los atributos arquitectónicos derivados de las alometrías específicas a cada zona, además de los atributos más clásicos de estructura y de composición pudieron calcularse y relacionarse con las variaciones espaciales de la biomasa y de las reservas de carbono. Esta tesis doctoral proporcionó nuevos datos para los futuros mapas de biomasa en el marco del seguimiento del carbono forestal en África Central.

Palabras clave: alometría de los árboles, altura total, dimensiones de la copa, biomasa aérea, reservas de carbono, especies coexistentes, tipo de bosque, rasgos o atributos arquitectónicos, rasgos funcionales, variación espacial de la biomasa, África Central.
Doi : $10.19182 / \mathrm{bft} 2020.343 .931853$

Droit d'auteur (C) 2020, Bois et Forêts des Tropiques ( Cirad Date de publication : 28 février 2020 
Bois et Forêts des Tropiques - ISSN: L-0006-579X

Volume $343-1^{\text {st }}$ quarter - January 2020 - p. 85-86 THESIS ABSTRACT

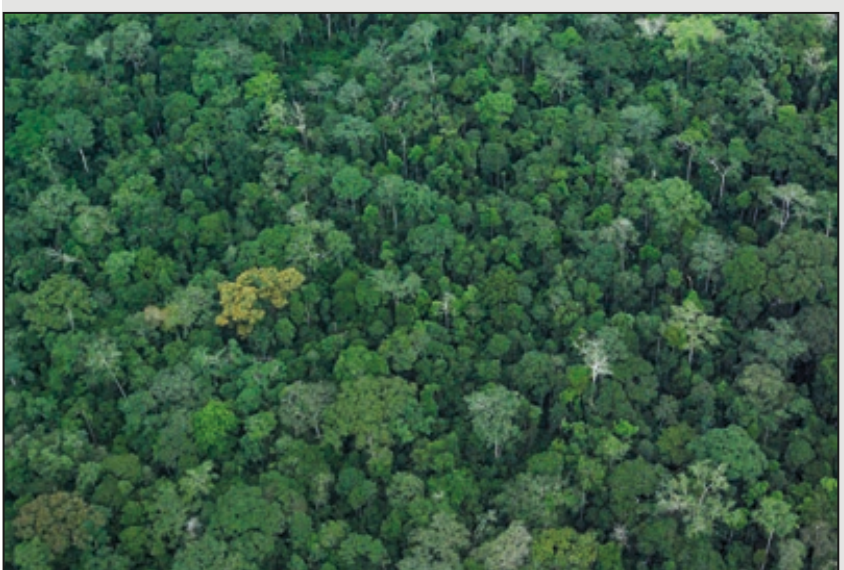

Photo 1.

Forêts tropicales d'Afrique centrale.

Photo J.-L. Doucet.

\section{Photo 1.}

Tropical forest in central Africa

Photo J.-L. Doucet.

Foto 1.

Bosques tropicales de África Central.

Foto J.-L. Doucet.

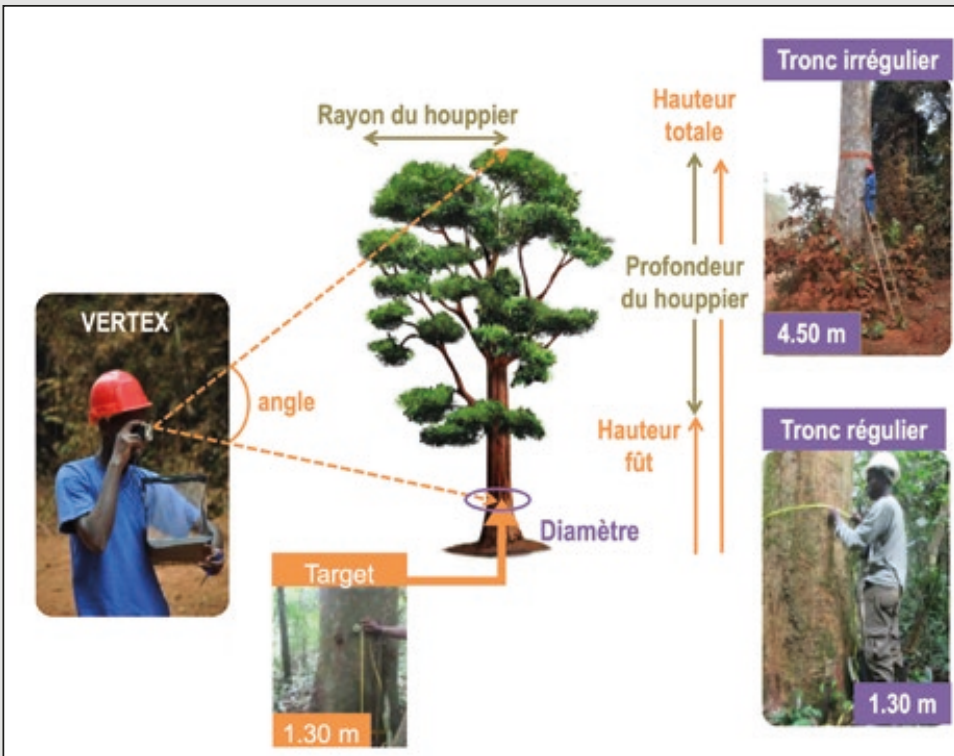

Figure 1.

Protocole de mesure des dimensions des arbres : mesure du diamètre à $1,30 \mathrm{~m}$ pour les arbres à tronc régulier et à $30 \mathrm{~cm}$ au-dessus des contreforts pour les arbres à tronc irrégulier, mesure de la hauteur totale et de la hauteur fût avec le VERTEX IV, et mesure des dimensions du houppier (rayon et profondeur).

Figure 1.

Tree measurement protocol: diameter measured at $1.30 \mathrm{~m}$ in height for trees with regular trunks, and $30 \mathrm{~cm}$ above the buttresses for trees with irregular trunks; total height and trunk height measured with VERTEX IV; measurement of crown dimensions (radius and depth). Translation of the captions of the figure: Angle: angle; Rayon du houppier: crown radius; Diamètre: diameter $(1.30 \mathrm{~m})$; Target: target $(1.30 \mathrm{~m})$; Hauteur totale: Total height; Profondeur du houppier: crown depth; Hauteur fût: trunk height; Tronc irrégulier: Irregular trunk (4.50 m); Tronc régulier: regular trunk.

Figura 1.

Protocolo de medida de las dimensiones de los árboles: medida del diámetro a 1,30 m para los árboles de tronco regulary a $30 \mathrm{~cm}$ por encima de los estribos para los árboles de tronco irregular, medida de la altura total y de la altura de fuste con el VERTEX IV, y medida de las dimensiones de la copa (radio y profundidad). Traducción de las leyendas de la figura: Angle: ángulo; Rayon du houppier: radio de la copa; Diamètre: diámetro; Target: objetivo; Hauteur totale: altura total; Profondeur du houppier: profundidad de la copa; Hauteur fût: altura del fuste; Tronc irrégulier: tronco irregular; Tronc régulier: tronco regular.

\section{Publications}

Loubota Panzou G. J., Doucet J.-L., Loumeto J. J., Biwole A., Bauwens S., Fayolle A., 2016. Biomasse et stocks de carbone des forêts tropicales africaines (synthèse bibliographique). Biotechnologie, Agronomie, Société et Environnement, 20 (4) : 508-522. https://popups.uliege.be/1780-4507/ index.php?id=13232

Fayolle A., Loubota Panzou G. J., Drouet T., Swaine M. D., Bauwens S., Vleminckx J., Biwole A., Lejeune P., Doucet J.-L., 2016. Taller trees, denser stands and greater biomass in semi-deciduous than in evergreen lowland central African forests. Forest Ecology and Management, 374: 42-50. https://doi.org/10.1016/i.foreco.2016.04.033

Loubota Panzou G. J., Fayolle A., Feldpausch T. R., Ligot G., Doucet J.-L., Forni E., Zombo I., Mazengue M., Loumeto J. J., Gourlet-Fleury S., 2018 What controls local-scale aboveground biomass variation in central Africa? Testing structural, composition and architectural attributes. Forest Ecology and Management, 429: 570-578. https://doi.org/10.1016/i.foreco.2018.07.056

Loubota Panzou G. J., Ligot G., Gourlet-Fleury S., Doucet J.-L., Forni E. Loumeto J. J., Fayolle A., 2018. Architectural differences associated with functional traits among 45 coexisting tree species in Central Africa. Functional Ecology, 32 (11): 2583-2593. https://doi.org/10.1111/13652435.13198 